\title{
Age-Dependent Change of Ro- kumigan, Hachimijiogan and Goshajinkigan-Induced Vasodila- tations in Rat Aorta
}

Keywords: Rokumigan; Hachimijiogan; Goshajinkigan; Vasodilatation; Endothelium

\begin{abstract}
Background: Rokumigan, hachimijiogan and goshajinkigan are traditional Kampo formulations. These formulations are considered to be useful for edema, water disturbance, and urinary problem. Rokumigan is composed of six kinds of crude herbal drugs. Hachimijiogan comprises the same six kinds of herbs and two crude drugs and goshajinkigan includes the same eight herbs of hachimijiogan and two others. We demonstrated that these formulations possess vasodilating actions in rat aorta. Traditionally, rokumigan is thought to be useful for young persons, while hachimijiogan and goshajinkigan are thought to be for elderly persons. In this study, we compare the age-dependen modulation of these Kampo formulations.

Method: Young wistar rats (10 to 15 week old) and aged rats (35 to 40 week old) were used to investigate the age-dependent actions of these formulations. The isolated aorta ring strips were fixed in Krebs-Henseleit solution. After the contractile response induced by norepinephrine became steady, Kampo formulations are added. Results: Rokumigan showed potent vasodilatation in young rats. However, the vasodilation - induced by rokumigan was attenuated in aged rats. On the contrary, hachimijiogan - induced vasodilatation in aged rats was to the same extent as that in young rats. Furthermore, compared with young rats, goshajinkigan - induced vasodilatation significantly increased in aged rats. Endothelium removal and L-NAME (NO synthase inhibitor) attenuated rokumigan, hachimijiogan and goshajinkigan - induced vasodilatation in young rats. However, endothelium removal and the pretreatment of L-NAME did not modify rokumigan - induced vasoditation in aged rats. Endothelium remova and L-NAME attenuated hachimijiogan and goshajinkigan - induced vasodilatation in aged rats.

Conclusion: Rokumigan - induced vasodilatation attenuated as age while the potency of hachimijiogan and goshajinkigan - induced vasodilatations was not changed or increased in aged rats compared with young rats. Our results suggested that the difference of age related dependency of kampo formulations on vasodilatation was derived from their different dependency to the endothelium.
\end{abstract}

\section{Introduction}

Rokumigan, hachimijiogan and goshajinkigan are traditional Japanese herbal (Kampo) formulations. They have been used for the patients suffering from urinary tract symptoms, chronic nephritis and low back pain [1]. The compositions of crude drugs in three kampo formulations are similar, but the number of crude drugs are different Rokumigan comprises six crude herbal drugs (Rahmanniae Radix, Corni Fuructus, Dioscorea Rhizoma, Alismatis Rhizoma, Poria cocos, and Moutan Cortex). Hachimijiogan comprises the six drugs of rokumigan and two additional crude drugs (Cinnamomi Cortex and Processi Aconiti Tuber) and goshajinkigan comprise the eight crude drugs of hachimijiogan and two additional crude drugs (Achyranthis Radix and Plantaginis Semen). The exact compositions of crude

\section{Journal of}

Integrative Medicine \& Therapy

\section{Seiichiro Nishida ${ }^{1^{*}}$, Katsuharu Tsuchida ${ }^{2}$ and Hiroyasu Satoh ${ }^{3}$}

${ }^{1}$ Koriyama Seiran Hospital, Yamatokoriyama, Japan

${ }^{2}$ Rational Medicinal Science, Doshisha Women's College, Kyotanabe, Japan

${ }^{3}$ Health Life Science, Shitennoji University, Habikino, Japan

\section{*Address for Correspondence}

Seiichiro Nishida, Koriyama Seiran Hospital, Yamatokoriyama, Nara Prefecture 639-1136, Japan, Tel: +81-0743-56-8000; Fax: +81-0743-59-0022; E-mail: drmaro@m4.kcn.ne.jp

\section{Submission: 31 October, 201 \\ Accepted: 20 December, 2016 \\ Published: 27 December, 2016}

Copyright: (๑) 2016 Nishida, et al. This is an open access article distributed under the Creative Commons Attribution License, which permits unrestricted use, distribution, and reproduction in any medium, provided the original work is properly cited.

drugs in three kampo formulations are summarized in Table 1 . We have shown the same summary of compositions in previous article entitled "comparative study of vasodilatation induced by rokumigan, hachimijiogan, and goshajinkigan in the Rat aorta" of this journal [2].

In Kampo medicine, rokumigan, hachimijiogan and goshajinkigan are considered to regulate "Jin" functions. The "Jin" has been considered to regulate the water balance of body, urinary track system, hearing system, bone and bone marrow [1,3]. Therefore, "Jin" can be explained as kidney and urinary track system. The failure of "Jin" directly leads to the incidences of edema or dehydration, frequent urination, osteoporosis, and also some dysfunctions related with ageing.

Table 1: The compositions of the crude drugs contained in rokumigan, hachimijiogan and goshajinkigan.

\begin{tabular}{|l|l|l|l|}
\hline & Rokumigan & Hachimijiogan & Goshajinkigan \\
\hline Rahmanniae Radix & 5.0 & 6.0 & 5.0 \\
\hline Corni Fuructus & 3.0 & 3.0 & 3.0 \\
\hline Dioscorea Rhizoma & 3.0 & 3.0 & 3.0 \\
\hline Alismatis Rhizoma & 3.0 & 3.0 & 3.0 \\
\hline Poriacocos & 3.0 & 3.0 & 3.0 \\
\hline Moutan Cortex & 3.0 & 3.0 & 3.0 \\
\hline Cinnamomi Cortex & 1.0 & & 1.0 \\
\hline Processi Aconiti Tuber & 0.5 & 1.0 & \\
\hline Achyranthis Radix & & 3.0 & \\
\hline Plantaginis Semen & 3.0 & & \\
\hline
\end{tabular}

Rokumigan (TJ-87), hachimijiogan (TJ-7) and goshajinkigan (TJ-107, Tsumura Co. Tokyo) were used. Values in this table means the contained gram (g) of crude drugs in kampo formulations. 
Citation: Nishida S, Tsuchida K, Satoh H, et al. Age-Dependent Change of Rokumigan, Hachimijiogan and Goshajinkigan-Induced Vasodilatations in Rat Aorta. J Integrative Med Ther. 2016;3(1): 6

\section{ISSN: $2378-1343$}

Rokumigan can regulate "Jin" functions, resulting in the improvement of body water balance that is exhausted by inflammatory disorder and unusual heat. Rokumigan is effective for not only children easily to develop a fever, but also adult persons. On the other hand, both hachimijiogan and goshajinkigan have been prescribed traditionally for elderly persons with the symptoms such as dry skin, dry mouth, weakening of legs, benign prostatic hypertrophy and lower back pain [1]. Both drugs have also been well known to improve frequent urination or lower urinary tract symptoms $[1,3]$ Hachimijiogan relaxes acetylcholine (ACh) - induced contraction of isolated rat bladder strips [4]. Goshajinkigan has been shown to beso effective for overactive bladder $(\mathrm{OAB})$ in male and female patients $[5,6]$. It has been reported that goshajinkigan regulate bladder activity via sympathetic and parasympathetic nervous systems and suppress C-fiber activation [7,8]. The indications of hachimijiogan and goshajinkigan are similar, but goshajinkigan is relatively useful for more elderly persons suffering from lower back pain and leg pain [1]. Furthermore, the other beneficial effect for elderly people by goshajinkigan has been reported. Sarcopenia is one of the age-related phenomena. Sarcopenia means the phenomenon that muscle mass and strength decrease as age. It has been reported that goshajinkigan protects against sarcopenia promotion in senescence-accelerated mice [9].

Rokumigan, hachimijiogan and goshajinkigan have similar indications; regulating edema and water balance. We ascribe partially these indications to the improvement of blood flow induced by these three Kampo formulations. We infer that these clinical indications are partially derived from the vasodilation induced by these Kampo formulations [2]. And these three Kampo formulations have same mechanisms of vasodilatation such as $\mathrm{Ca}^{2+}$ channel inhibition, $\beta$-receptor activation and producing nitric oxide(NO) from endothelium [2].

We have investigated the age-dependent modulation of the vasodilating effects induced by herbal medicine and Kampo formulations. Aging produces various deleterious physiological and histological changes in vascular system such as a plaque formation and wall thickening [10]. Endothelial dysfunction induced by aging is mainly associated with decreased production of NO, resulting in impaired vasodilatation [11]. In aged endothelial cells, NO synthesis decreased [11]. Loss of PI3K/Akt-dependent eNOS phosphorylation is one of the mechanisms explaining the reduction of eNOS activity in old rats [12]. Furthermore, it was reported that age-related change of endothelium-dependent vasodilatation is also involved with reduced bioavailability of NO and response of smooth muscle to NO [11]. Alteration of endothelium-dependent vasodilatation is characterized by reduced agonist-induced vasodilatation. Acetylcholine (Ach) induced vasodilatation was attenuated in aorta and mesenteric artery of elderly rats due to malfunction of endothelium [13-15]. And sheer stress - induced vasodilatation (which is response of endothelium to shear stress resulting the production of NO) is also impaired by aging [16].

However, we have reported that herbal medicine or Kampo medicine showed the tendency that they exhibit more potent vasodilatation in elder rats than in young rats $[17,18]$.

Ginkgo biloba extract (GBE) is one of the famous herbal medicines [19]. GBE is effective for ischemic brain injury and cerebral disorders with aging. GBE induces dose-dependent vasodilatation [19]. Bilobalide, one of the main constituents in GBE, also exhibits dose-dependent vasodilatation on rat aorta [19]. Bilobalide-induced vasodiltation is attenuated as age develop ( 5 to 25 week old rats) [17] Ginkgo biloba extract-induced vasodilatation is also modulated by aging. In 5 to 20 week old rats, GBE-induced vasodilatation (at 0.01 $\sim 1 \mathrm{mg} / \mathrm{ml}$ ) decreased with age. However, in 25 week old rats, GBE induced vasodilatation (at $0.01 \sim 1 \mathrm{mg} / \mathrm{ml}$ ) reversely enhanced [17]. The age-dependent attenuation of bilobalide - induced vasodilatation was more severe than that of GBE - induced vasodilatation [17]. The GBE, as a mixture of some constituents, kept vasodilatation potency in elderly rats. However, in 25 week old rats, the vasodilatations induced by bilobalide were severely attenuated [17]. We infer that the interaction between some constituents in GBE preserve GBE induced vasodilatations from age - dependent change.

Mokuboito, one of the Kampo formulations, comprises four crude drugs [20]. Mokuboito is useful for edema, dyspnea caused by heart failure [20]. Mokuboito possesses dose - dependent vasodilating actions. Sinomenine acutum is one of the crude herbs in Mokuboito. The extract of Sinomenine acutum also exhibits vasodilatation on rat aorta [18]. Sinomenine is one of the constituents in Mokuboito. This alkaloid is consisted in Sinomenine acutum. Sinomenine has potent vasodilating actions $[17,18]$. However, sinomenine - induced vasodilating actions severely attenuated in elder rats (35 to 65 week old rats) [18]. The extract of Sinomenin actum was also attenuated in elder rats ( 35 to 65 week old rats). However, the attenuation of Sinomenine acutum - induced vasodilatation is milder than that of sinomenine [18]. The extract of Sinomenine acutum is a mixture of some constituents. Therefore, Sinomenine acutum is resistant to the influence of age. On the other hand, the vasodilatation induced by Mokuboito did not attenuate in elder rats. Mokuboito comprises four crude drugs and is more resistant to aging than Sinomenine acutum or sinomenine [18]. We infer that the interaction with herbal drugs and the ingredients in Mokuboito enhances vasodilating effects and prevent the age - dependent attenuation of pharmacological responses.

Rokumigan has been traditionally thought to be suitable for young persons, while hachimijiogan and goshajinkigan have been prescribed for elderly persons $[1,3]$. We infer that the difference of agedependency between Kampo formulations is partially derived from the age - dependent changes of vasodilatations induced by Kampo medicines. Therefore, we compared the vasodilating effects induced by Kampo formulations in young and elder rats and investigate the age - dependent alterations through by using rat aortic ring strips.

\section{Materials and Methods}

All experiments were carried out according to the guidelines laid down by the Nara Medical University Animal Welfare Committee and the Doshisha Women's Collegeanimal Welfare Committee.

Male Wistar rats were used in this study. To investigate the age dependent modulation of pharmacological response, young rats (10 to 15 week old) and aged rats ( 35 to 40 week old) were used. The rats were anesthetized with ether or isoflurane, and were euthanized by exsanguinations. The thoracic aorta was quickly removed, and was cut into rings of $3 \mathrm{~mm}$ in length. The experiments were carried 
Citation: Nishida S, Tsuchida K, Satoh H, et al. Age-Dependent Change of Rokumigan, Hachimijiogan and Goshajinkigan-Induced Vasodilatations in Rat Aorta. J Integrative Med Ther. 2016;3(1): 6.

out as reported previously $[2,17,18,20]$. The vascular rings were suspended between lower and upper triangular - shaped stainless steel stirrups in a jacketed organ chamber filled with $20 \mathrm{ml}$ modified Krebs - Henseleit solution. The lower stirrup was anchored and the upper stirrup was attached to a force - displacement transducer (Nihon Kohden TB-652T, Tokyo, Japan) to record the isometric force. All rings were stretched to generate a resting tension of $1.2 \mathrm{~g}$. The modified Krebs - Henseleit solution was comprised of $118 \mathrm{NaCl}$, $4.6 \mathrm{KCl}, 1.2 \mathrm{MgSO}_{4}, 1.2 \mathrm{KH}_{2} \mathrm{PO}_{4}, 11.1$ glucose, 27.2 $\mathrm{NaHCO}_{3}, 0.03$ $\mathrm{Na}_{2}$ - ethylnediaminetetraacetic acid (EGTA), and $1.8 \mathrm{mM} \mathrm{CaCl}_{2}$. The chamber solution was kept at $37{ }^{\circ} \mathrm{C}$ and oxygenated with $95 \%$ $\mathrm{O}_{2}$ and $5 \% \mathrm{CO}_{2}$. After $40 \mathrm{~min}$ of resting, $5 \mu \mathrm{M}$ norepinephrine (NE) was added to the tissue bath. After the contractile response became steady, the drugs were cumulatively administrated into the bath solution. The effects of the drugs were measured 10-20 min after the responses became steady. The relaxation responses were calculated as the percentage decrease compared with the maximal contraction induced by NE. Endothelium was carefully removed by rubbing internal lumen with smooth and thin plastic sticks. The absence of functional endothelium was confirmed by a lack of the relaxant response to acetylcholine (ACh, $1 \mu \mathrm{M}$ ).

Rokumigan (TJ-87), hachimijiogan (TJ-7) and goshajinkigan (TJ-107) are produced by Tsumura Co. (Tokyo, Japan) for clinical use in Japan. They are a spray - dried powder extracted with boiling water of a mixture of medical herbs. The drugs used in this study are supplied by Tsumura Co. The components of three formulations are summarized in Table 1.The Kampo formulations were dissolved with DMSO. And $N^{\mathrm{G}}$-Nitro-L-arginine methyl ester (L-NAME) (Wako Chemical, Osaka, Japan) were used. $\mathrm{NaCl}, \mathrm{KCl}, \mathrm{MgSO}_{4}, \mathrm{KH}_{2} \mathrm{PO}_{4}$, glucose, $\mathrm{NaHCO}_{3}, \mathrm{CaCl}_{2}$, and DMSO were also purchased from Wako Chemical. NE (Daiichi Sankyo Co., Tokyo, Japan) was used. All values are represented as the means \pm S.E.M. The differences of data in mean values were analyzed by analysis of variance (ANOVA) and a $\mathrm{p}$ value of less than 0.05 was considered significant.
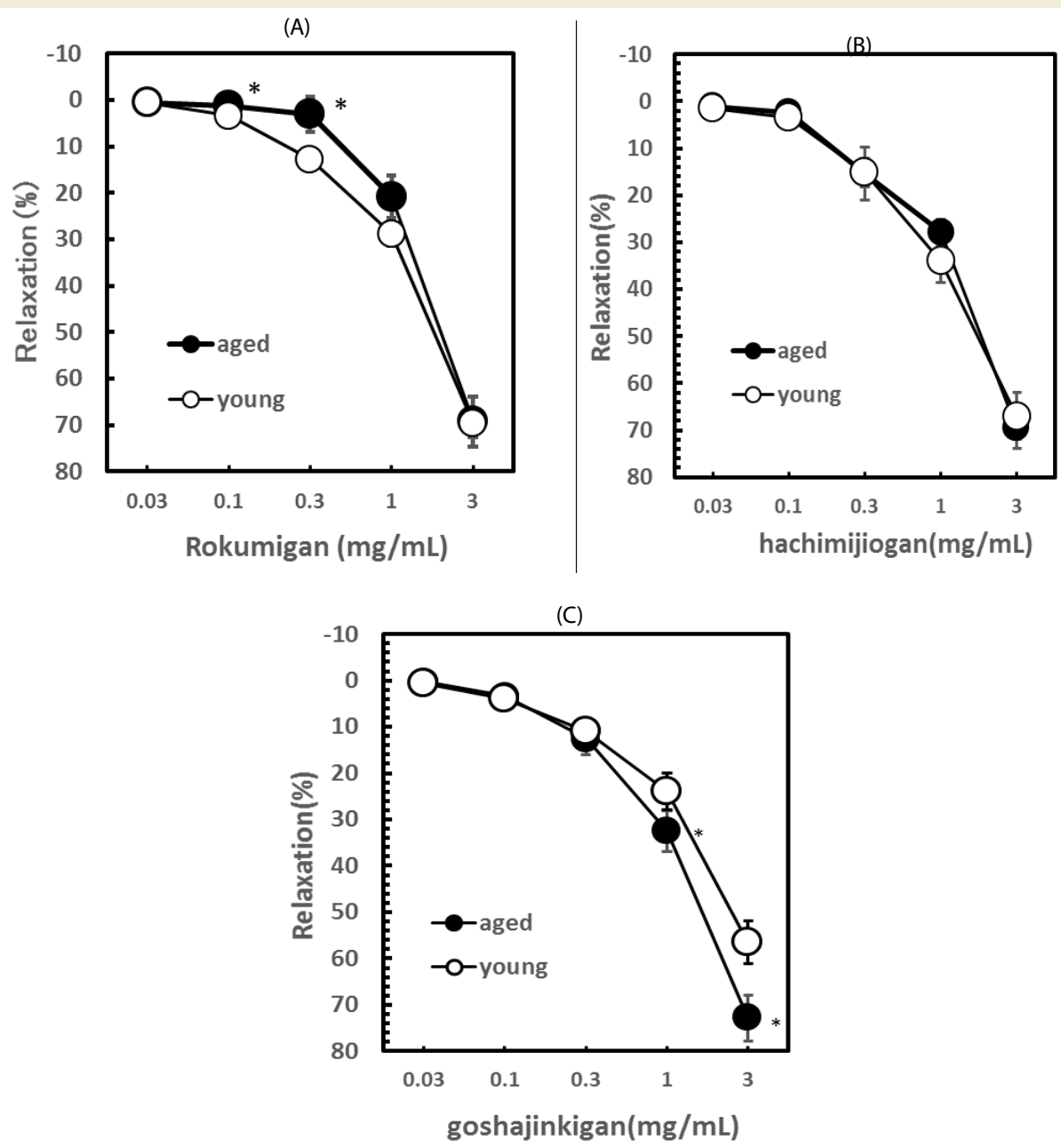

Figure 1: Age dependent change of vasodilatations induced by rokumigan, hachimijiogan and goshajinkigan.(A) Modulation of rokumigan - induced vasodilatation. (B) Modulation of hachimijiogan - induced vasodilatation. (C) Modulation of goshajinkigan - induced vasodilatation.Symbols are: Open circles: control (youngrats) $(n=8)$ and Close circles: aged rats $(n=8)$. Values $(\%)$ represent mean \pm S.E.M.

${ }^{*} \mathrm{P}<0.05$ : significantly different from control. 
Citation: Nishida S, Tsuchida K, Satoh H, et al. Age-Dependent Change of Rokumigan, Hachimijiogan and Goshajinkigan-Induced Vasodilatations in Rat Aorta. J Integrative Med Ther. 2016;3(1): 6.

ISSN: $2378-1343$

\section{Results}

Age-dependent change of vasodilatations induced by rokumigan, hachimijiogan and goshajinkigan

Application of NE caused remarkable vasoconstriction of resting aorta. We have reported that rokumigan, hachimijiogan and goshajinkigan caused the vasodilatations in a concentrationdependent manner in young rats (10 to 15 week old rats) [2].

Rokumigan at $0.03 \mathrm{mg} / \mathrm{ml}$ had less vasodilatation (by 0.51 $\pm 0.25 \%$ ), butit caused significant vasodilatation at over $0.1 \mathrm{mg} / \mathrm{mL}$; by $69.3 \pm 3.9 \%(\mathrm{n}=8, \mathrm{p}<0.01)$ at $3 \mathrm{mg} / \mathrm{mL}$ in young rats. In aged rats (35 to 40 week old rats), the vasodilatation induced by rokumigan was also observed. At lower concentration, the potency of rokumigan - induced vasodilatations tended to decrease in aged rats, compared with those in young rats. Rokumigan - induced vasodilatations were attenuated significantly in aged rats at $0.1,0.3 \mathrm{mg} / \mathrm{mL}$ (Figure $1 \mathrm{~A}$ ). At $3 \mathrm{mg} / \mathrm{mL}$, rokumigan sustained almost the same potency as young rats. Hachimijiogan at $0.1-3 \mathrm{mg} / \mathrm{mL}$ also caused the significant vasodilatation in young rats; by $67.0 \pm 4.9 \%(\mathrm{n}=8, \mathrm{p}<0.01)$ at $3 \mathrm{mg} /$ $\mathrm{mL}$. There are not significant differences in hachimijiogan -induced vasodilatations between young rats and aged rats (Figure 1B).

On the contrary, goshajinkigan induced more potent vasodilatation in aged rats, compared with young rats. Goshajinkigan induced stronger vasodilatations at 1.0 and $3.0 \mathrm{mg} / \mathrm{ml}$ in aged rats than those in young rats significantly (Figure 1C). The percent of vasodilatations in young rats was $24.0 \pm 4.0(\mathrm{n}=8)$ and $58.1 \pm 2.9 \%$ $(\mathrm{n}=8)$ at 1.0 and $3.0 \mathrm{mg} / \mathrm{mL}$, respectively. Whereas the percent of vasodilatations in aged rats was32.4 $\pm 4.4(\mathrm{n}=8, \mathrm{p}<0.05)$ and $72.8 \pm$ $4.9 \%(\mathrm{n}=8, \mathrm{p}<0.05)$ at 1.0 and $3.0 \mathrm{mg} / \mathrm{mL}$, respectively.

Age-dependent modulation of endothelium-dependent vasodilatations induced by rokumigan, hachimijiogan and goshajinkigan

To investigate the endothelium dependency of vasodilatations, L-NAME $(100 \mu \mathrm{M})$ and endothelium removal were pretreated. In young rats, we have been reported that L-NAME and endothelium removal attenuated kampo formulations-induced vasodilatations in previous reports [2]. However, in aged rats, L-NAME and endothelium removal did not attenuate rokumigan-induced vasodilatations at 0.03 - $1.0 \mathrm{mg} / \mathrm{mL}$ (Table 2). Only at $3 \mathrm{mg} / \mathrm{mL}$, endothelium removal and L-NAME alter the rokumigan - induced vasodilatation.
We reported that the hachimijiogan - and the goshajinkigan - induced vasodilatations were also reduced by pretreatments with both L-NAME and endothelium removal [2]. The endothelium removal decreased the vasodilatations induced by hachimijiogan at $0.3-3.0 \mathrm{mg} / \mathrm{mL}$ in young rats [2]. L-NAME also reduced the degree of vasodilatations at $1.0 \mathrm{mg} / \mathrm{ml}$ and at $3.0 \mathrm{mg} / \mathrm{ml}$ of hachimijiogan in young rats [2]. In aged rats, L-NAME and endothelium - removal also significantly attenuated hachimijiogan - induced vasodilatations at $0.3,1.0$ and $3.0 \mathrm{mg} / \mathrm{mL}$. Endothelium - removal decreased the vasodilatations; $6.8 \pm 3.0 \%(\mathrm{n}=6, \mathrm{p}<0.05)$ at $0.3 \mathrm{mg} / \mathrm{mL}, 16.0 \pm 7.2$ $\%(\mathrm{n}=6, \mathrm{p}<0.05)$ at $1.0 \mathrm{mg} / \mathrm{mL}, 53.3 \pm 3.6 \%(\mathrm{n}=6, \mathrm{p}<0.05)$ at 3.0 $\mathrm{mg} / \mathrm{mL}$ in aged rats.

As well in young rats, the goshajinkigan $(3.0 \mathrm{mg} / \mathrm{mL})$ - induced vasodilatation decreased by $43.7 \pm 1.3 \%(\mathrm{n}=8, \mathrm{p}<0.01)$ in the presence of L-NAME, and by $47.6 \pm 3.9 \%(\mathrm{n}=8, \mathrm{p}<0.01)$ under the condition of endothelium-removal [2]. In aged rats, L-NAME and endothelium - removal also significantly attenuated goshajinkigan induced vasodilatations at $0.3 \sim 3.0 \mathrm{mg} / \mathrm{mL}$ (Table 2). In aged rats, endothelium - removal significantly decreased the goshajinkigan induced vasodilatations; $20.0 \pm 5.2 \%(\mathrm{n}=6, \mathrm{p}<0.05)$ at $1.0 \mathrm{mg} /$ $\mathrm{mL}, 53.0 \pm 3.2 \%(\mathrm{n}=6, \mathrm{p}<0.01)$ at $3.0 \mathrm{mg} / \mathrm{mL}$ (Table 2$)$. L-NAME also significantly attenuated goshajinkigan - induced vasodilatations in aged rats (Table 2). These results indicate that goshajinkigan elicit endothelium - dependent vasodilatation in aged rats.

\section{Discussion}

The present experiments have shown that (a) rokumigan, hachimijiogan and goshajinkigan dilated rat aorta constricted by $\mathrm{NE}$ in a concentration-dependent manner, (b) rokumiganinduced vasodilatation was attenuated in aged rats (35 to 40 week old rats), (c) endothelium removal and L-NAME did not attenuate rokumigan - induced vasodilatation in aged rats, (d) hachimijiogan - induced vasodilatation did not change significantly in aged rats, (e) goshajinkigan - induced vasodilatation was potentiated in aged rats, and (f) the endothelium - dependencies of hachimijiogan and goshajinkigan - induced vasodilatation are stronger in aged rats than that of rokumigan - induced vasodilatation.

Rokumigan, hachimijiogan and goshajinkigan have various clinical usages. The effectiveness for edema and coldness is considered to be partially due to the vasodilating effects. We infer that the resultant vasodilating effects of these Kampo formulations might be associated

Table 2: Endothelium dependency of the vasodilatations induced by rokumigan, hachimijiogan and goshajinkigan in aged rats.

\begin{tabular}{|c|c|c|c|c|c|c|c|}
\hline & & $\mathbf{N}$ & $0.03 \mathrm{mg} / \mathrm{ml}$ & $0.1 \mathrm{mg} / \mathrm{ml}$ & $0.3 \mathrm{mg} / \mathrm{ml}$ & $1.0 \mathrm{mg} / \mathrm{ml}$ & $3.0 \mathrm{mg} / \mathrm{ml}$ \\
\hline \multirow{3}{*}{ Rokumigan } & Control & 8 & $0.51 \pm 0.20$ & $1.2 \pm 0.80$ & $3.8 \pm 3.0$ & $20.8 \pm 4.5$ & $69.3 \pm 5.4$ \\
\hline & Endothelium (-) & 6 & $0.51 \pm 0.20$ & $1.2 \pm 0.80$ & $1.0 \pm 0.86$ & $18.1 \pm 4.0$ & $54.4 \pm 5.1^{*}$ \\
\hline & L-NAME & 6 & $0.0 \pm 0.0$ & $0.47 \pm 0.24$ & $5.0 \pm 0.67$ & $17.8 \pm 1.5$ & $55.3 \pm 2.3^{*}$ \\
\hline \multirow{3}{*}{ Hachijiogan } & Control & 8 & $1.0 \pm 0.67$ & $2.4 \pm 1.2$ & $15.4 \pm 5.6$ & $27.8 \pm 2.7$ & $69.6 \pm 4.2$ \\
\hline & Endothelium (-) & 6 & $0.0 \pm 0.0$ & $1.3 \pm 1.0$ & $6.8 \pm 3.0^{*}$ & $16.0 \pm 7.2^{*}$ & $53.3 \pm 3.6^{* *}$ \\
\hline & L-NAME & 6 & $0.0 \pm 0.0$ & $0.0 \pm 0.0$ & $2.7 \pm 1.3^{*}$ & $11.8 \pm 1.0^{*}$ & $47.0 \pm 2.2^{* *}$ \\
\hline \multirow{3}{*}{ Goshajinkigan } & Control & 8 & $0.40 \pm 0.20$ & $3.4 \pm 1.5$ & $12.5 \pm 3.4$ & $32.4 \pm 4.4$ & $72.8 \pm 4.9$ \\
\hline & Endothelium (-) & 6 & $0.0 \pm 0.0$ & $2.8 \pm 1.4$ & $9.5 \pm 3.3$ & $20.0 \pm 5.2^{*}$ & $53.0 \pm 3.3^{* *}$ \\
\hline & L-NAME & 6 & $0.12 \pm 0.11$ & $2.1 \pm 1.0^{*}$ & $4.9 \pm 2.0^{*}$ & $21.8 \pm 5.2^{*}$ & $52.8 \pm 6.4^{* *}$ \\
\hline
\end{tabular}

Controls are vasodilatations in aged rats (35 to 40 week old rats). Values (\%) represent Mean \pm S.E.M.

$*: p<0.05$.

$* *: p<0.01$

Symbols of ${ }^{*}$ and ${ }^{* *}$ mean a significance compared with the vasodilating effect of control at each concentration 
Citation: Nishida S, Tsuchida K, Satoh H, et al. Age-Dependent Change of Rokumigan, Hachimijiogan and Goshajinkigan-Induced Vasodilatations in Rat Aorta. J Integrative Med Ther. 2016;3(1): 6.

with the multiple other pharmacological actions. The vasodilatations would improve peripheral blood flow, and then warm extremities and ameliorate peripheral edema [21,22]. Moreover, rokumigan and hachimijioga has been shown the extensive usefulness for memory enhancement, precognitive ability, prevention of neuronal degeneration, and protection from ischemia/reperfusion-induced renal injury [23-26].

In our previous paper, although there are some differences among the components of three kampo formulations, the profiles of vasodilatations were almost similar in young rats [2]. However, in traditional Kampo medicine, rokumigan is thought to be suitable for young persons, while hachimijiogan and goshajinkigan are useful for elderly persons. We infer that the difference of age-dependency on effectiveness between Kampo formulations is partially derived from the age - dependent changes of vasodilatations induced by Kampo medicines. In our study, rokumigan - induced vasodilatation was attenuated in aged rats. Furthermore, in aged rats, L-NAME and endothelium removal failed to alter the rokumigan - induced vasodilatation at 0.03 to $1.0 \mathrm{mg} / \mathrm{mL}$, except for $3.0 \mathrm{mg} / \mathrm{mL}$. These results indicate that rokumigan has weak potency of eliciting endothelium -dependent relaxation in aged rats.

On the contrary, hachimijiogan - induced vasodilatation did not significantly alter in aged rats. And L-NAME and endothelium removal attenuated the hachimijiogan - induced vasodilatation. This result indicates that endothelium - dependent vasodilatation is retained in aged rats.

Furthermore, goshajinkigan - induced vasodilatation was stronger in aged - rats than in young rats. Endothelium - removal and L-NAME significantly attenuated goshajinkigan - induced vasodilatation in aged rats. These results also indicate that endothelium dependency of goshajinkigan - induced vasodilatation is also retained in aged rats. Under the endothelium - removal, goshajinkigan - induced vasodilatations at $3.0 \mathrm{mg} / \mathrm{ml}$ were $42.9 \pm 4.6(\mathrm{n}=6)$ in young rats and $53.8 \pm 3.3(\mathrm{n}=6, \mathrm{p}<0.05)$ in aged rats. Although the detailed mechanism is not evident, the potency of goshajinkigan-induced vasodilatation without endothelium in aged rats was stronger than that in young rats. Therefore, these results indicate that goshajinkigan might enhance both endothelium dependent - and independent vasodilatation in aged rats.

Hachimijiogan and goshajinkigan have shown endothelium dependent vasodilatation in aged rats more markedly than rokumigan. Goshajinkigan showed more potent vasodilatation in aged rats than in young rats. The reason why endothelium dependencies were different between Kampo formulations in aged rats is not yet clear.

Aging process of endothelium function have been elucidated by many studies $[10,11,12,27]$. In aged endothelial cells, NO synthesis decreased $[11,12,27]$. Akt enhances eNOS activity by phosphorylation [28]. Attenuation of PI3K/Akt-dependent eNOS phosphorylation is one of the mechanisms explaining the reduction of eNOS activity in old rats [12]. And reduced bioavailability of $\mathrm{NO}$ and response of smooth muscle to NO are also involved in endothelial dysfunction $[11,27]$. It has been reported that augmentation of oxidative stress with aging induces the reduction of NO bioavailability and endothelial dysfunction [11,27]. Direct measurement of eNOS activity can be determined by western blot analysis method [28] And confirmation of NO formation can be performed by electron resonance spectroscopy [28,29]. Aged endothelial cells can be obtained from aged animal arteries using pretreatment of collagenase and cell culture technique [28]. Direct evaluation of eNOS activity and NO production would convince that Kampo formulations induce endothelium dependent vasodilatation and NO production. Furthermore, direct measurement of eNOS activity and NO production in aged endothelial cells would reveal the mechanisms or process by which Kampo formulations activate aged endothelial cells. Further studies are needed to elucidate detailed mechanism by which Kampo formulations activate aged endothelium. Anyway, hachimijiogan and goshajinkigan is well adapted to aged rat aorta due to sustaining and enhancing endothelium function. And these results might be one of the reasons why hachimijiogan and goshajinkigan are suitable for elderly people. Hachimijiogan and goshajinkigan might induce stronger vasodilatations in elderly persons than young persons.

Compared with basic formulation of rokuimigan, hachimijiogan and goshajinkigan are added some herbs (Table 1). And the ingredients in the added herbs might alter the vasodilating potency of hachimijiogan and goshajinkigan. Cinnamomi Cortex and Processi Aconiti Tuber are added in hachimijiogan and goshajinkigan. They also possess the potent vasodilating action. Cinnamaldehyde, one of the ingredients in Cinnamomi cortex, exerts the vasodilating actions via endothelium - derived relaxation factor (NO) and by blocking $\mathrm{Ca}^{2+}$ channel [30]. Mesaconitine, main ingredient, in Aconiti tuber increases the intracellular $\mathrm{Ca}^{2+}$ concentration $\left(\left[\mathrm{Ca}^{2+}\right]_{\mathrm{i}}\right)$ in endothelium cell. The $\left[\mathrm{Ca}^{2+}\right]_{i}$ increase activates the production of $\mathrm{NO}$ and then, the vasodilatation is produced [31]. In rat mesenteric artery, mesaconitine causes the vasodilatation mediated through the endothelium -derived hyperpolarization factor (EDHF) [32].These herbs might strengthen the vasodilation induced by hachimijiogan and goshajinkigan. Therefore, it is also important to investigate the combined effects of Cinnamomi cortex and Aconiti tuber induced vasodilatations.

Hachimijiogan maintained the potency of total vasodilatation and its endothelium - dependency in aged rats. However, goshajinkigan showed enhanced vasodilatations in aged rats. Achyranthis Radix and Plantaginis Semen are added in goshajinkigan. However, these two herbs are not known yet whether they possess vasodilating effects or not. We infer the interaction of ingredients in goshajinkigan generate the enhanced potency of vasodilatation. Further studies are needed to elucidate how the individual herbs or ingredients contributes to the total pharmacological effects of Kampo formulations.

The clinical effective concentrations of their constituents and ingredients (phytochemicals) in these Kampo formulations are not sufficiently known yet. There is no report for the serum concentrations of these Kampo formulations at all after oral intake, presumably because so many ingredients exist in a crude drug and a formulation. The concentrations of formulations used in this study would be higher than the clinical concentrations obtained after oral administration. In general, the concentrations in vitro experiments are relatively higher, since the pharmacological responses would be observed as acute responses within the limited time in addition to 
Citation: Nishida S, Tsuchida K, Satoh H, et al. Age-Dependent Change of Rokumigan, Hachimijiogan and Goshajinkigan-Induced Vasodilatations in Rat Aorta. J Integrative Med Ther. 2016;3(1): 6.

the low sensitivities of the in vitro preparations. To elucidate more comprehensive understandings about the clinical contribution of these Kampo formulations, further in vivo experiments are needed.

In conclusion, rokumigan, hachimijiogan and goshajinkigan have the vasodilating effects. The vasodilatations are modified by aging. Rokumigan-induced vasodilatation was attenuated in aged rats. Hachimijiogan sustained the potency of vasodilatation in aged rats. And goshajinkigan enhanced vasodilating actions in aged rats. The vasodilatations by three Kampo formulations showed the different endothelium dependency in aged rats. Hachimijiogan and goshajinkigan kept endothelium - dependent vasodilatations in aged rats, while endothelium - dependent actions of rokumigan was attenuated in aged rats. We infer that maintained potency of hachimijiogan and goshajinkigan would make these two formulations suitable for elderly persons.

Finally, these Kampo formulations can produce the plenty pharmacological actions as well as the vasodilatation. To elucidate more comprehensive understandings about the clinical contribution of these Kampo formulations, further studies are needed in various age.

\section{References}

1. Sato Y, Hanawa T, Arai M, Cyong J, Fukuzawa M, et al. (2005) Introduction to Kampo - Japanese traditional medicine. Elsevier, Tokyo, Japan.

2. Nishida S, Tsuchida K, Satoh H (2015) Comparative study of vasodilatation induced by rokumigan, hachimijiogan, and goshajinkigan in the rat aorta. $J$ Integrative Med Ther 2: 6

3. Yoshiharu S, Wu J (1997) Kampo treatment for climateric disorders: a hand book for practioners. Paradigm Publications, Massachusetts, USA.

4. Ito Y, Seki M, Nishioka Y, Kimura M, Yasuda A, et al. (2009) Pharmacological effects of Hachi-mi-jio-gan extract (Harncare) on the contractile response and on pharmacologically relevant receptors in the rat bladder. Yakugaku Zasshi 129: 957-964.

5. Ogushi T, Takahashi S (2007) Effect of Chinese herbal medicine on overactive bladder. Hinyokika Kiyo 53: 857-862.

6. Kajiwara M, Mutaguchi K (2008) Clinical efficacy and tolerability of goshajinki-gan, Japanese traditional herbal medicine, in females with overactive bladder. Hinyokika Kiyo 54: 95-99.

7. Nishijima S, Sugaya K, Miyazato M, Ogawa Y (2007) Effect of Gosha-jinkigan, a blended herbal medicine, on bladder activity in rats. J Urol 177: 762 765.

8. Imamura T, Ishizuka O, AizawaN, Zhong C, Ogawa T, et al. (2008) Goshajinki-gan reduces transmitter proteins and sensory receptors associated with $\mathrm{C}$ fiber activation induced by acetic acid in rat urinary bladder. Neurourol Urodyn 27: 832-837.

9. Kishida Y, Kagawa S, Arimitsu J, Nakanishi M, Sakashita N, et al. (2015) Gosha-jinki-gan (GJG), a traditional Japanese herbal medicine, protects agains sarcopenia in senescence-accelerated mice. Phytomedicine 22: 16-22.

10. Gaballa MA, Jacob CT, Raya TE, Liu J, Simon B, et al. (1998) Large artery remodeling during aging: biaxial passive and active stiffness. Hypertension 32: $437-443$.

11. Payne JA, Reckelhoff JF, Khalil RA (2003) Role of oxidative stress in agerelated reduction of NO-cGMP-mediated vascular relaxation in SHR. Am J Physiol Regul Integr Comp Physiol 285: R542-R551.

12. Smith AR, Hagen TM (2003) Vascular endothelial dysfunction in aging: loss of Akt-dependent endothelial nitric oxide synthase phosphorylation and partial restoration by $(\mathrm{R})$-alpha-lipoic acid. Biochem Soc Trans 31: 1447-1449.

13. Miyata N, Tsuchida K, Okuyama S, Otomo S, Kamata K, et al. (1992) Agerelated changes in endothelium-dependent relaxation in aorta from genetically diabetic WBN/Kob rats. Am J Physiol 262: H1104-H1109.
14. Kim SY, Park JT, Park JK, Lee JS, Choi JC (2011) Aging impairs vasodilatory responses in rats. Korean $\mathrm{J}$ Anesthesiol 61: 506-510.

15. Atkinson J, Tatchum-Talom R, Capdeville-Atkinson C (1994) Reduction of endothelial function with age in the mesenteric arterial bed of the normotensive rat. $\mathrm{Br} \mathrm{J}$ Pharmacol 111: 1184-1188.

16. Sun D, Huang A, Yan EH, Wu Z, Yan C, et al. (2004) Reduced release of nitric oxide to shear stress in mesenteric arteries of aged rats. Am J Physiol Heart Circ Physiol 286: H2249-H2256.

17. Nishida S, Satoh H (2005) Age-related changes in the vasodilating actions of Ginkgo biloba extract and its main constituent, bilobalide, in rat aorta. Clin Chim Acta 354: 141-146.

18. Satoh H, Nishida S (2012) Lack of the age-dependent alteration in mokuboito (mu-fang-yi-tang). J US-China Med Sci 9: 1-8.

19. Satoh H, Nishida S (2004) Electropharmacological actions of Ginkgo biloba extract on vascular smooth and heart muscles. Clin Chim Acta 342: 13-22.

20. NishidaS, Satoh H (2007) Vascular pharmacology of mokuboito (mu-fang-yitang) and its constituents on the smooth muscle and the endothelium in rat aorta. Evid Based Complement Alternat Med 4: 335-341.

21. Isobe H, Yamamoto K, Cyong JC (2003) Effects of hachimi-jio-gan (ba-weidi-huang-wan) on blood flow in the human central retinal artery. Am $\mathrm{J}$ Chin Med 31: 425-435

22. Suzuki Y, Goto K, Ishige A, Komatsu Y, Kamei J (1998) Effects of Goshajinki-gan, a kampo medicine, on peripheral tissue blood flow in streptozocininduced diabetic rats. Methods Find Exp Clin Pharmacol 20: 321-328.

23. Park E, Kang M, Oh JW, Jung M, Park C, et al. (2005) Yukmijihwang-tang derivatives enhance cognitive processing in normal young adults: a doubleblinded, placebo-controlled trial. Am J Chin Med 33: 107-115.

24. Iwasaki K, Kobayashi S, ChimuraY, Taguchi M, Inoue K, et al. (2004) A randomized, double-blind, placebo-controlled clinical trial of the Chinese herbal medicine "ba wei di huang wan" in the treatment of dementia. J Am Geriatr Soc 52: 1518-1521.

25. Rho S, Kang M, Choi B, Sim D, Lee J, et al. (2005) Effects of Yukmijihwangtang derivatives (YMJd), a memory enhancing herbal extract, on the geneexpression profile in the rat hippocampus. Biol Pharm Bull 28: 87-93.

26. Kang DG, Sohn EJ, Moon MK, Mun YJ, Woo WH, et al. (2006) Yukmijihwangtang ameliorates ischemia/reperfusion-induced renal injury in rats. J Ethnopharmacol 104: 47-53.

27. Donate AJ, Morgan RG, Walker AE, Lesniewski LA (2015) Cellular and molecular biology of aging endothelial cells. J Mol Cell Cardiol 89: 122-135

28. Anselm E, Chataigneau M, Nidaye M, Chataigneau T, Schini-Kerth VB (2007) Grape juice causes endothelium-dependent relaxation via a redox-sensitive Src- and Akt-dependent activation of eNOS. Cardiovasc Res 73: 404-413.

29. Kleschyov AL, Munzel T (2002) Advanced spin trapping of vascular nitric oxide using colloid iron diethyldithiocarbamate. Methods Enzymol 359: 42-51.

30. Yanaga A, Goto H, Nakagawa T, Hikiami H, Shibahara N, et al. (2006) Cinnamaldehyde induces endothelium-dependent and -independent vasorelaxant action on isolated rat aorta. Biol Pharm Bull 29: 2415-2418.

31. Mitamura M, Horie S, Sakaguchi M, Someya A, Tsuchiya S, et al. (2002) Mesaconitine-induced relaxation in rat aorta: involvement of $\mathrm{Ca}^{2+}$ influx and nitric-oxide synthase in the endothelium. Eur J Pharmacol 436: 217-225.

32. Mitamura M, Boussery K, Horie S, Murayama T, Van de Voorde J (2002) Vasorelaxing effect of mesaconitine, an alkaloid from Aconitum japonicum, on rat small gastric artery: possible involvement of endothelium-derived hyperpolarizing factor. Jpn J Pharmacol 89: 380-387.

\section{Acknowledgements}

Authors wish to thank for supply of the kampo formulations, rokumigan (TJ-87), hachimijiogan (TJ-7) and goshajinkigan (TJ107), from Tsumura Co. 